\title{
Reflections on TEAP: training for the future of medical physics
}

\author{
Anne Perkins
}

Published online: 9 June 2013

(C) Australasian College of Physical Scientists and Engineers in Medicine 2013

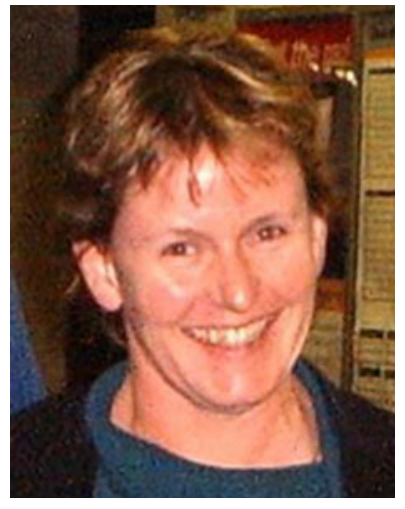

2013 marks the tenth anniversary of the ACPSEM's Training, Education and Assessment Program (TEAP) for radiation oncology medical physics. It also marks the launch of TEAP for diagnostic imaging medical physics, a new program that caters for both nuclear medicine and radiology medical physics trainees. For both these reasons, this seems like a good time to stop and reflect on the importance of training for medical physics in Australia and New Zealand. What has been achieved? What is the purpose of TEAP? And where do we need to go next?

I believe that TEAP marks a fundamental shift in our view of what it means to be a medical physics professional. When I first started working in medical physics in the late 1980s, there was very little formal training. Medical physics was mostly learnt on the job. The skills learnt were dictated by the clinical needs of the department, very often by being thrown in at the deep end. Many highly skilled

A. Perkins $(\bowtie)$

ACPSEM, Suite 7.12, 247 Coward Street, Mascot,

NSW 2020, Australia

e-mail: Anne.Perkins@acpsem.org.au medical physicists learnt their trade in this system, but the level of expertise that developed was variable. Those who wished to do so could seek external recognition through the ACPSEM accreditation examinations for radiation oncology medical physics, nuclear medicine physics and radiology medical physics. But in most cases achieving accreditation through these processes was not required for professional advancement.

Now the story is very different. TEAP registrars commit to completing a highly structured training program which aims to develop the knowledge, skills and attitudes to practice as a medical physics professional. Registrars must undertake an extensive period of competency based training in a hospital, which is far broader in scope than the previous accreditation programs. Radiation oncology medical physics registrars must achieve competencies in treatment planning, brachytherapy and ancillary modules that were not part of the previous system. Diagnostic imaging registrars must undertake common modules which address core professional skills, regardless of the discipline in which they intend to specialize. In addition to the competency based training, registrars in both programs must also demonstrate skills in research and communication by completing an accredited postgraduate degree in medical physics, publications and conference presentations. Competency is assessed by clinical supervisors in departments during the training period, and assessed again by the certification panels during final examinations. Completion of TEAP results in the award of ACPSEM Certification as a Medical Physics Specialist, and is recognized by employers and governments as the new standard for professional competence.

In the 10 years since the introduction of TEAP for radiation oncology medical physics, the program has become well established. There are approximately 100 registrars 
enrolled in TEAP at 33 accredited clinical departments across Australia and New Zealand. More than 40 registrars have successfully completed TEAP and achieved certification. This is a huge achievement and something of which the ACPSEM should be proud. Lack of government funding for the diagnostic imaging medical physics program has caused delays in implementation, but the first registrars should be able to enrol later in 2013.

While we've got something to be proud of in the progress we've made with TEAP in the past decade, it doesn't come without a cost. TEAP is a demanding program, typically requiring between 3 and 5 years to complete. Standards are high, and there is a huge amount to get through. This is tough not only on registrars but also on the clinical departments that support them. In the 10 years since TEAP was introduced, departments have had to learn how to provide a structured training program that covers all the TEAP requirements, and to provide the resources to do this. Most medical physicists are not trained educators and have had to develop new skills to enable them to provide effective clinical supervision. The challenges are exacerbated when departments are short staffed and struggling to cope with the clinical workload.

Understanding the requirements of the training program is a significant challenge for a department taking on their first TEAP registrars. One of the most frequent complaints about TEAP is that the expected standard is not clear. What is the desired end point of the program? Are we looking to produce an expert who can practise as an independent medical physicist in sole practice, or are we looking for a person who can operate effectively as part of a team?

For the radiation oncology TEAP, I think it is now becoming clear that the end point for TEAP is different from the previous accreditation system. The old Accreditation in Radiotherapy Equipment Commissioning and Quality Assurance (ARECQA) examination expected that the candidate could act as an independent practitioner able to work without supervision in the relatively narrow areas of radiation safety, dosimetry, equipment commissioning and quality assurance. The TEAP examinations are assessing a registrar to act as qualified physicist who can work as part of a team under minimal supervision but in a much broader area. This difference in expectations is reflected in the preambles to the questions used in the final practical examinations. For ARECQA, the preamble read: "You are a consultant physicist called into this hospital to..." For TEAP candidates, the wording is changed to read: "Your chief physicist has asked you to undertake the following task..." Whereas ARECQA candidates were expected to be able to deal with unfamiliar equipment and to respond appropriately to differences in protocols and procedures in different hospitals, TEAP candidates are expected to be familiar with equipment, procedures and protocols in their own department. A TEAP registrar is not necessarily expected to have all the answers to every conceivable problem at their fingertips, but should demonstrate that they can practice safely. Recognizing the limitations of one's own knowledge and formulating appropriate strategies in response is one of the keys to safe practice.

A frequent complaint about TEAP is that the program requirements are too onerous, making it difficult for registrars to complete all the program requirements in the expected timeframe. I believe that in many cases this difficulty is not so much a fundamental problem with TEAP but is more due to a lack of training support. In the early days of TEAP, expectations were not well understood by registrars, supervisors or by clinical departments, and many of the support structures that now exist were not in place. Registrars were sometimes left to flounder without the support of an experienced clinical supervisor or a TEAP coordinator/preceptor and without receiving external feedback on their progress. Slow progress in these circumstances is perhaps understandable. Individual motivation also has a strong influence on a registrar's rate of progress. For registrars employed in permanent positions there are generally no negative consequences if TEAP completion is slow. For registrars on temporary contracts in supernumerary training positions, completing TEAP before the contract runs out is a very strong motivating factor.

So is TEAP too onerous? For those of us with a role to play in managing and developing TEAP, this is very challenging question. On the one hand, there is an understandable desire from members of the profession that the TEAP should include everything that a registrar might need to learn to help them in their future practice. Technology, imaging and therapy techniques are constantly evolving and TEAP should equip registrars to deal with a rapidly changing field. On the other hand it's clear that we can't just keep adding new topics to TEAP syllabus indefinitely.

To give a radiotherapy example, should we insist that every registrar has been fully trained in how to commission volumetric modulated arc therapy (VMAT), now rapidly becoming a standard treatment technique? I would argue against this. I believe that every registrar should demonstrate that they understand what is required to commission a new treatment technique, not necessarily VMAT. Having acquired this knowledge, they will then be able to apply similar principles to safely commission VMAT or any other new technique. Equipping registrars with generic skills that will enable them to practice safely is far more important than having a detailed knowledge of any particular technique. After all, one of the key attributes that we can offer as medical physicists is the ability to apply scientific thinking to solve new clinical problems.

Many of you will be aware that the Clinical Training Guide for the radiation oncology TEAP is currently being revised module by module. As each module is considered, 
questions like the one I raised about VMAT commissioning must be considered. Should this topic be included? Can something else be omitted as it is no longer relevant? As the diagnostic imaging medical physics TEAP gets off the ground this year, similar questions about the course content will be sure to arise.

The answer to questions like these should come from the medical physics profession as a whole, not just from one or two individuals. I urge all ACPSEM members to get involved in contributing to improving TEAP. Take the opportunity to learn more about TEAP and provide feedback to the certification panels if you have concerns or suggestions. Send in a response when policies and training documents are circulated for comment. The more input our members provide, the more likely it is that our TEAP programs will meet the expectations of our profession. After all, what could be more important to our profession than training the workforce of the future? 